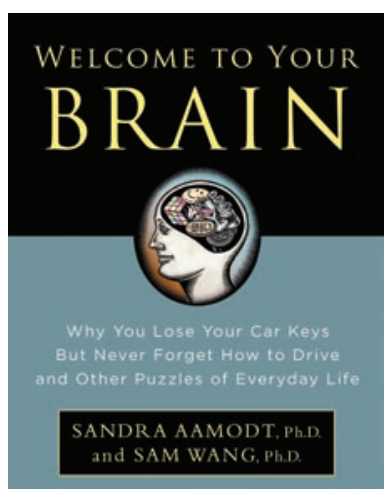

\section{Welcome to your brain}

\author{
Why you lose your car keys but never forget how to drive \\ and other puzzles of everyday life
}

Sandra Aamodt and Sam Wang

Bloomsbury USA. New York, New York, USA. 2008.

240 pp. \$24.95. ISBN: 978-1-59691-283-0 (hardcover).

\section{Reviewed by Joshua Tate Dudman}

Janelia Farm Research Campus, Howard Hughes Medical Institute, Ashburn, Virginia, USA.

E-mail: dudmanj@janelia.hhmi.org
W illiam James originally published The principles of psychology in 1890 (1), a culmination of years of research that summarized the state of knowledge in the nascent field of psychology. An early chapter is devoted to "Habit," a reflection of James's belief that the most important aspect of brain function is the basic tendency of our nervous system to form stimulus-response associations based upon past experience. James admonished his reader to "make our nervous system our ally instead of our enemy" by understanding and exploiting this principle of brain function. In a similar spirit of pragmatic demystification, Sandra Aamodt and Sam Wang have written Welcome to your brain, an owner's manual for the engine between our ears.

Aamodt and Wang draw upon a wealth of experience in basic research in neuroscience. Aamodt became an editor at a leading journal in the field, Nature Neuroscience, following her doctoral and postdoctoral work studying molecular signaling during the development of the auditory and visual systems. Wang is currently an associate professor of neuroscience at Princeton University and has published papers on topics ranging from synaptic plasticity to the evolution of brain structure. Through these complementary backgrounds, and presumably wide-ranging personal interests, the authors bring an admirable breadth of knowledge to their first book. The product is an entertaining collection of detailed anecdotes from a wide range of topics in neuroscience and a coherent synthesis of disparate research fields for a general audience.

Welcome to your brain loosely borrows the organization typical of many neuroscience textbooks, beginning with basic cellular function and building toward an understanding of how interacting networks of neurons produce sensation and behavior. It is always challenging to assess the appropriate level of detail to provide in everything from teaching an undergraduate course to writing a general text. Cellular neuroscience and the physiological properties of neurons make this challenge more difficult because they are far removed from the experiences of the average reader. At times, the early chapters felt hurried, and the introduction to basic concepts was a bit disappointing, especially coming from two neuroscientists with a background in physiology.

Once the subject moves on to sensory systems, the book picks up steam and finds a rhythm as a readable and entertaining text. There is enough explanation for a reader with little or no prior science background to learn about how the brain works. A great strength of the book is that it contains enough detailed asides for readers with a more extensive background in science to enrich their understanding of those everyday experiences subjected here to detailed biological explanation. The authors' conversational, humorous, and intimate voice wavers somewhat, but often gives the feel of an informed tour guide who favors common sense and reasonable explanations over excessive demonstrations of erudition - a Garrison Keillor for the neuroscience crowd.

Like a good owner's manual, the coverage is as close to exhaustive as one might hope for in a concise text. However, as is also true of a manual, a reader with avid interest will at times be disappointed by the lack of depth. Of particular frustration is the omission of a reference list. This choice may reflect the authors' desire to create an introductory text that is not intimidating, yet other authors of popular neuroscience texts (David Linden and Antonio Damasio come to mind) provide well-organized reference sections. Such lists not only provide a service to the reader, but also lend credibility to the authors. A skeptical reader may rely upon such demonstrations of credibility in a text that spends a good deal of time debunking popular misconceptions about scientific results. Indeed, by failing to direct the reader to primary research, the authors risk the possibility that some of the anecdotes and speculation in this book will lead to misconceptions in need of their own debunking.

The bookshelf of popular neuroscience texts is beginning to runneth over. This collection can tend toward myopathy as the admirable motive to write about what one knows best leads to a text that reduces the brain to a single fundamental function. Welcome to your brain represents a refreshing departure from this trend. It provides a wide-ranging introduction to the brain that does an excellent job of summarizing our current understanding and providing a sober assessment of the places where research falls short. I suspect, however, that our debt to the book will not be to the compendium of interesting details, but rather to the authors' belief that a little education about brain function can help one think more clearly about debates that cross scientific lines into social policy. Their restatement of the nature versus nurture debate, for example, as a question of using social policy to create an environment that maximizes genetic potential is incisive and provides something missing from an owner's manual: insight.

1. James, W. 1971. Principles of psychology. 19th edition. Encyclopedia Britannica Inc. Chicago, Illinois, USA. 897 pp. 